All letters are subject to editing and may be shortened. Letters should be sent to the BJGP office by e-mail in the first instance, addressed to

journal@rcgp.org.uk (please include your postal address). Alternatively, they may be sent by post as an MS Word or plain text version on CD or DVD. We regret that we cannot notify authors regarding publication. Letters not published in the Journal may be posted online on our Discussion Forum. For instructions please visit: http://www.rcgp.org.uk/bjgp-discuss

\section{GPs' attitudes to hypertension guidelines}

\section{Heneghan and colleagues used an} internet survey in 2006 to assess GPs' awareness, agreement and adherence to hypertension guidelines. ${ }^{1}$ They found $51 \%$ of responders knew that blood pressure (BP) based on home/self monitoring should be adjusted downwards by $10 / 5 \mathrm{mmHg}$. However, as highlighted in Carlsen and colleagues' meta-synthesis showing GPs questioning guidelines, only $5 \%$ said they had adopted this particular hypertension guideline. ${ }^{2}$

These findings are reflected in our more qualitative telephone survey of GPs conducted in 2007. We decided to ask GPs their opinions about the home BP monitoring trial, after posting information about the trial and the BHS/NICE guidelines to them. Of the $40 \mathrm{GPs}$ contacted, 34 responded ( $85 \%$ response rate). The majority of responders were male (74\%). The mean number of call attempts made to contact a GP was 1.9. We found that $50 \%$ (17/34) of GPs knew the correct target for home BP monitoring. Interestingly, 88\% $(30 / 34)$ of GPs had received the information but only $40 \%$ of these $(12 / 30)$ had read the information. Even fewer, seven GPs (21\%), had understood the aims of the trial. We also found that $85 \%(29 / 34)$ of GPs were in favour of home monitors as they felt they eliminated white coat hypertension and provided a true reflection of BP. One GP strongly disagreed with home BP monitors as he felt they were inaccurate and caused problems for patients and GPs. There was a small proportion (four GPs) that had mixed views about monitors as they felt they caused patient anxiety. In our survey, two GPs were unhappy to cooperate with the trial in aiming for the home BP target.
One GP felt the target was too low and predisposed his patient to falls and the other felt that he was not well informed about the trial and was therefore not willing to cooperate. The GPs in our survey emphasised that they were often 'too busy and had no time' to read the information sent to them. Therefore they felt the telephone call was an important method of relaying important information about the trial and the home BP target to them. In a trial such as ours, the role of the GP is pivotal in achieving target BP and changing treatment, therefore education of the GP is paramount.

Our findings support those of Heneghan. Not only do GPs 'not have time to read and memorise all the guidance', but 'they may not adopt the recommendations despite high awareness.'

\section{Sandeep Takher}

Foundation Year Two Doctor, Community Health Sciences, St George's, University of London. E-mail: skt00@doctors.org.uk

\section{Gillian Kyei}

Speciality Training Year One Doctor, Community Health Sciences, St George's, University of London

\section{Pippa Oakeshott}

Research GP, Community Health Sciences, St George's, University of London

\section{Sally Kerry}

Principal Investigator, Community Health Sciences, St George's, University of London

\section{Acknowledgements}

Funding was provided by the Stroke Association and The Isaac Shapira Trust.

\section{REFERENCES}

1. Heneghan C, Perera R, Mant D, Glasziou P Hypertension guideline recommendations in general practice: awareness, agreement, adoption and adherence. Br J Gen Pract 2007; 57(545): 948-952.
2. Carlsen B, Genton C, Pope C. Thou shalt versus thou shalt not: a meta-synthesis of GPs' attitudes to clinical practice guidelines. Br J Gen Pract 2007; 57(545): 971-978

DOI: 10.3399/bjgp08X279788

\section{Minor surgery in general practice: are sterilised gloves necessary?}

The use of non-sterile gloves during minor surgery is normal care in general practice. The Dutch College of General Practitioners has issued a guideline for infection prevention based on the regulation of the Dutch Working Party on Infection Prevention. ${ }^{1}$ Under the section 'Minor surgery' it is stated: 'When a wound is closed with a suture, one has to work under sterile conditions. This means: sterile gloves, sterile working area and sterile material.' There is no evidence in the guideline to support this recommendation.

A retrospective study of the records of patients undergoing minor surgical procedures was performed. Records were searched for surgical site infection (SSI). SSI is defined according to the guideline for the prevention of SSI. ${ }^{2}$

All surgical procedures were performed in an examination room in a general practice. After washing the hands with a disinfecting soap the gloves were put on. The skin was disinfected. A sterile dressing, steam-heated sterilised instruments and pre-packaged sterilised gauzes were used. The wound was closed using nylon or resolvable sutures which were removed after 7-14 days.

One hundred and sixty-eight minor surgical procedures were performed on 133 patients. Sutures were used in 131 procedures. Five procedures were 
excluded from this analysis because sterile gloves were used. In the remaining 126 procedures there were four complications of which three (2.4\%) were a SSI. None of these patients had diabetes or used anticoagulant or antiplatelet medication. In the group in whom no sutures were used there were no complications.

Cultures on blood agar plates were taken of clean examination gloves after opening a new box and 1 week later. They showed no bacterial growth. It might be concluded that bacterial contamination of the gloves occurs after they are put on. This corresponds with earlier findings. ${ }^{3}$

Randomised controlled trial's on this subject are scarce because of ethical constraints. ${ }^{4}$ One study showed that the risk for wound infection in dermatological surgery after using clean gloves is not significantly greater than after using sterile gloves; that is, $1.7 \%$ versus $1.6 \% .^{5}$ This corresponds with our results showing that the use of clean gloves has a low incidence of SSI.

Despite the retrospective nature of our study, our results question the recommended routine use of sterile gloves for minor surgery in general practice but further research is needed. Based on earlier reports and the results of our study, the ethical considerations preventing a randomised controlled trial might be overcome.

\section{Marco L Bruens}

Trainee GP, Department of General Practice, Erasmus Medical Center, P.O. Box 2040, 3000 CA, Rotterdam, the Netherlands.

E-mail: M.Bruens@erasmusmc.nl

\section{Pieter J van den Berg}

Senior Lecturer, Department of General Practice, Erasmus Medical Center, 3000 CA, Rotterdam, the Netherlands,

\section{Jeroen MG Keijman}

Department of Medical Microbiology, Albert Schweitzer Hospital, 3300 AK Dordrecht, the Netherlands.

\section{REFERENCES}

1. http://www.wip.nl/free_content/Richtlijnen/ 1Huisartsen.pdf. (accessed 5 Mar 2008).
2. Mangram AJ, Horan TC, Pearson ML, et al. Guideline for prevention of surgical site infection, 1999. Hospital Infection Control Practices Advisory Committee. Infect Control Hosp Epidemiol 1999: 20(4): 250-278.

3. Luckey JB, Barfield RD, Eleazor PD. Bacterial count compriarisons on examination gloves from freshly opened boxes versus nearly empty boxes and from examination gloves before treatment versus after dental dam isolation. J Endod 2006; 32(7): 646-648

4. Sage DK, Argall J. Use of sterile gloves in the treatment of simple wounds. Emerg Med J 2003; 20(1): 66 .

5. Rogues AM, Lasheras A, Amici JM, et al. Infection control practices and infectious complications in dermatological surgery. J Hosp Infect 2007; 65(3): 258-263.

DOI: 10.3399/bjgp08X279797

\section{Centralised pathology services}

GPs frequently find high serum potassium results unexpected, difficult to explain and therefore hard to interpret. The need for serum potassium to be urgently repeated causes anxiety and frustration to both patient and GP. Usually no explanation for the spuriously high serum potassium concentration (pseudohyperkalaemia) is found, but our informal local survey suggested around $50 \%$ of GPs thought a laboratory error was the cause.

Our study ${ }^{1}$ showed that pseudohyperkalaemia is almost invariably associated with factors affecting the pre- analytical stage. This includes the phlebotomy and transport temperature of blood samples to the laboratory. We carried out an audit of all GP requested potassium results over a 4-year period (224 000 samples). We confirmed the findings of other workers ${ }^{2}$ that there is an inverse relationship between mean serum potassium concentration for the primary care population and outdoor ambient temperature. The proportion of results above the normal range $(5.2 \mathrm{mmol} / \mathrm{L}$ or higher) varied between $6 \%$ in hot weather to $17 \%$ in the coolest. This is partly due to passive movement of potassium from the intracellular to extracellular fluid as the energy dependant sodium/potassium pump activity declines as temperature falls. We reduced this frequency to between $4.5 \%$ and $9 \%$ (depending on external ambient temperature) by ensuring that phlebotomists did not facilitate venesection by asking patients to hand grip/fist clench. Potassium is released from myocytes into extracellular fluid during muscle contraction.

The majority of our ambulant GP patients attend hospital clinics for phlebotomy, all within 5 miles of the laboratory. Inter-site transport ensures that most samples arrive within 1 hour. However, proposed centralisation of pathology services (with laboratory closures) will increase sample transport

\section{Centralised pathology services:}

Figure 1. Effect of temperature and phlebotomy on the incidence of hyperkalaemia.

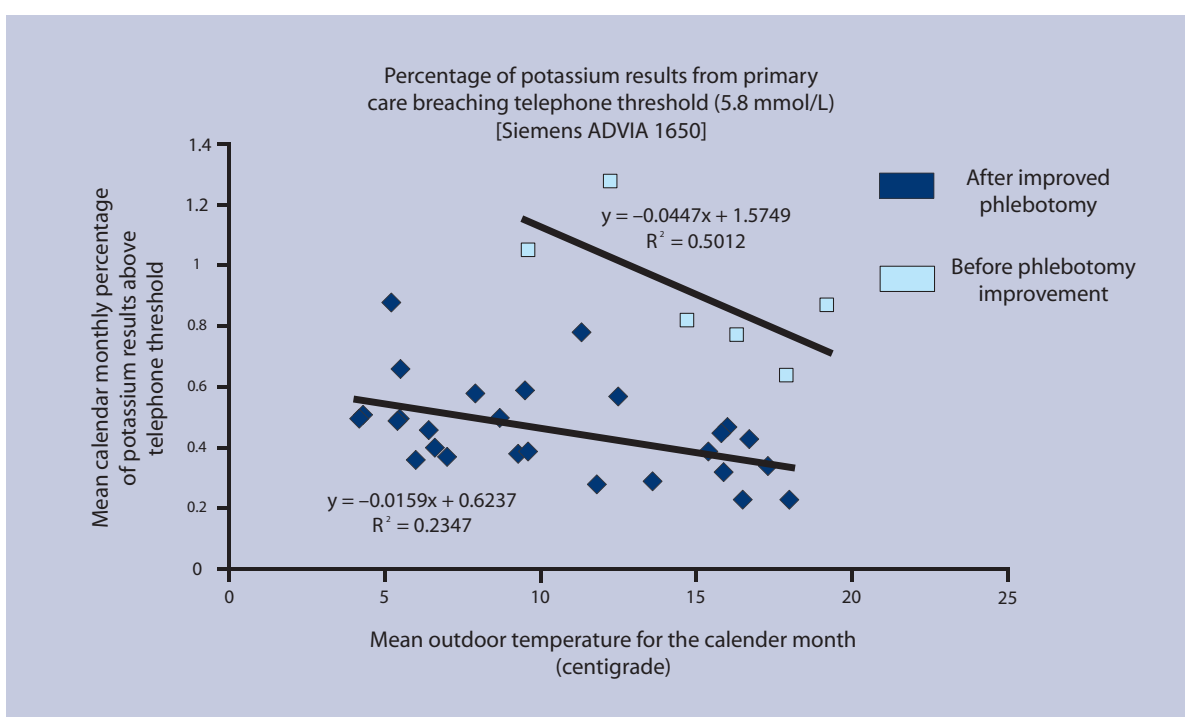

